MATHEMATICS OF COMPUTATION

Volume 75, Number 253, Pages 385-393

S 0025-5718(05)01776-X

Article electronically published on September 12, 2005

\title{
THE TRACE OF TOTALLY POSITIVE ALGEBRAIC INTEGERS
}

\author{
JULIÁN AGUIRRE, MIKEL BILBAO, AND JUAN CARLOS PERAL
}

ABSTRACT. For all totally positive algebraic numbers $\alpha$ except a finite number of explicit exceptions, the following inequality holds:

$$
\frac{1}{d}\left(\alpha_{1}+\cdots+\alpha_{d}\right)>\max \left(1.780022,1.66+\alpha_{1}\right),
$$

where $d$ is the degree of $\alpha$ and $0<\alpha_{1}<\cdots<\alpha_{d}$ its conjugates. This improves previous results of Smyth, Flammang and Rhin.

\section{INTRODUCTION AND MAIN RESULT}

Let $\alpha$ be a totally positive algebraic number of degree $d$, and let $0<\alpha_{1}<\cdots<$ $\alpha_{d}$ be its conjugates. Define the mean trace of $\alpha$ as

$$
\operatorname{tr}(\alpha)=\frac{1}{d} \sum_{i=1}^{d} \alpha_{i}
$$

For each odd prime $p, \theta_{p}=4 \cos ^{2}(\pi / p)$ is a totally positive algebraic integer of degree $(p-1) / 2$ such that $\operatorname{tr}\left(\theta_{p}\right)=2(p-2) /(p-1)$. Thus, 2 is a limit point of the set

$$
\mathcal{T}=\{\operatorname{tr}(\alpha): \alpha \text { is a totally positive algebraic integer }\} .
$$

It is an open question whether it is the smallest limit point, that is, whether $(0,2) \cap \mathcal{T}$ is a discrete set. One way to try to answer this question is to find lower bounds for the mean trace of totally positive algebraic integers. Previous such bounds, holding for all but finitely many explicit exceptions, are

$$
\begin{array}{ll}
\sqrt{e}, & \text { Schur (1918), } \\
1.733, & \text { Siegel (1945), } \\
1.7719, & \text { Smyth (1984), } \\
1.6+\alpha_{1}, & \text { Flammang, Rhin, Smyth (1997). }
\end{array}
$$

See [Si, Sm2, FRS]. In the last paper the authors state that it should be possible to improve the constant 1.6 in the last inequality to at least 1.65. We go a little bit further and prove that the inequality is valid with 1.66, and at the same time we obtain a new lower bound on the smallest limit point of $\mathcal{T}$.

Theorem. For all totally positive algebraic numbers $\alpha$, except a finite number of explicit exceptions, the following inequalities hold:

$$
\begin{aligned}
\operatorname{tr}(\alpha) & >1.780022, \\
\operatorname{tr}(\alpha) & >1.66+\alpha_{1} .
\end{aligned}
$$

Received by the editor July 2, 2004 and, in revised form, October 27, 2004.

2000 Mathematics Subject Classification. Primary 11R06, 11-04.

(C)2005 American Mathematical Society 
The idea of the proof is quite simple, and is based on the principle of auxiliary functions as in $[\mathrm{Sm} 2]$. For each $z \geq 0$ find a polynomial $Q \in \mathbb{Z}[x]$ and constants $a>0, b$ such that

$$
x-a \log |Q(x)| \geq b \quad \forall x>z .
$$

Averaging (3) over the conjugates of a totally positive algebraic integer $\alpha$ with minimal polynomial $P$ and $\alpha_{1} \geq z$ yields

$$
\operatorname{tr}(\alpha) \geq b+a \sum_{i=1}^{\partial \alpha} \log \left|Q\left(\alpha_{i}\right)\right|=b+a \log |\operatorname{Resultant}(P, Q)| .
$$

If $Q(\alpha) \neq 0$, then the resultant of $P$ and $Q$ is a nonzero integer, and it follows that $\operatorname{tr}(\alpha) \geq b$. For instance, if $Q(x)=x^{7}(x-1)^{9}$ and $a=1 / 10$, an easy calculation shows that $x-a \log |Q(x)|>1.46$ for all $x>0$, and hence $\operatorname{tr}(\alpha)>1.46$ for all totally positive algebraic integers except $\alpha=1$. In order to prove inequalities (11) and (2), we shall use different (and more complicated) polynomials $Q$, which turn out to be connected t o the so-called integer Chebyshev polynomials.

Given a positive integer $d$, let $\mathcal{Z}_{d}$ be the set of all nonzero polynomials with integer coefficients of degree less than or equal to $d$, and let $I \subset \mathbb{R}$ be a bounded, closed interval. A polynomial $P_{d} \in \mathcal{Z}_{d}$ such that

$$
\sup _{x \in I}\left|P_{d}(x)\right|=\inf _{p \in \mathcal{Z}_{d}} \sup _{x \in I}|p(x)|
$$

is called an integer Chebyshev polynomial or a polynomial of minimal diophantine deviation from zero in $I$. Moreover, $\lim _{d \rightarrow \infty} \sup _{x \in I}\left|P_{d}(x)\right|^{1 / d}=t_{\mathbb{Z}}(I)$ exists, and is called the integer transfinite diameter of $I$. A lower bound on the trace of totally positive algebraic integers was obtained in $\mathrm{BE}$ from estimates on $t_{\mathbb{Z}}([0,1 / m])$, $m \in \mathbb{N}$, while in FRS] inequalities such as (11) and (2) are used to get estimates on $t_{\mathbb{Z}}(I)$ for small intervals $I$.

The polynomials $Q$ used in our computations have a structure similar to that of the integer Chebyshev polynomials in [0,1]. Aparicio [A] showed that for sufficiently large $d, P_{d}$ has a factor of the form $x^{e_{1}}(1-x)^{e_{2}}(2 x-1)^{e_{3}}\left(5 x^{2}-5 x+1\right)^{e_{4}}$ with the exponents $e_{i}$ growing linearly with $d$. Borwein and Erdélyi [BE] found six more irreducible polynomials that are factors of all $P_{d}$ provided $d$ is large enough. The roots of all these factors are real and in the interval $[0,1]$. Habsieger and Salvy $\left[\mathrm{HS}\right.$ ] determined $P_{d}$ for $1 \leq d \leq 75$ and found a new irreducible factor which surprisingly has complex roots.

For each $z \geq 0$, we fix a finite set of irreducible polynomials $\mathcal{P}(z) \subset \mathbb{Z}[x]$ and let

$$
Q=\prod_{q \in \mathcal{P}(z)} q^{e_{q}}, \quad e_{q} \in \mathbb{N}
$$

This leads to the following optimization problem: maximize the function

$$
\Lambda\left(a_{q} \mid q \in \mathcal{P}(z)\right)=\inf _{x>z}\left(x-\sum_{q \in \mathcal{P}(z)} a_{q} \log |q(x)|\right)
$$

over all $a_{q}>0, q \in \mathcal{P}(z)$. There are heuristic rules to determine the polynomials in $\mathcal{P}(z)$ :

(1) All its roots should be positive. This is in accordance with the fact that all known irreducible factors of integer Chebyshev polynomials in $[0,1]$, with the one exception noted above, have their roots in $[0,1]$. 
(2) If $\beta$ is the smallest (positive) root of $q \in \mathcal{P}(z)$, then $\beta$ should be close to $z$.

(3) With $\beta$ as above, $\operatorname{tr}(\beta)-\beta$ should be as small as possible. The reason for this is the following: if we want to prove the inequality $\operatorname{tr}(\alpha)<\alpha_{1}+\gamma$, then all polynomials such that $\operatorname{tr}(\beta)<\beta+\gamma$ must be factors of the polynomial $Q$.

In all, we use 91 polynomials $Q_{j}, 1 \leq j \leq 91$, listed in Table 1 Thirty of them are among the 36 used in FRS. The rest were selected using the above heuristic criteria from a collection found by an exhaustive search. All of them are monic and have all their roots in the interval $(0, \infty)$, except for $Q_{38}$, which has two complex roots and corresponds to the polynomial $A_{8}$ in [HS after a suitable change of variable.

On the other hand, by a result of J.P. Serre (see the Note Added in Proof in $[\mathrm{Sm} 3]$ ), if $Q \in \mathbb{Z}[x], a>0$ and $b \in \mathbb{R}$ are such that

$$
x-a \log |Q(x)|>b \quad \forall x>0,
$$

then $b \leq 1.898302$. One immediate consequence is the impossibility of proving by this method that 2 is the smallest limit point of $\mathcal{T}$.

\section{Proof of the MAIN RESUlt}

In this section we prove the theorem stated in the Introduction. First of all, we remark that by considering $\alpha-\left\lfloor\alpha_{1}\right\rfloor$, we may assume that $0<\alpha_{1}<1$. Given $z \geq 0$, let $I(z) \subset\{1, \ldots, 91\}$ be the set of indexes such that $\mathcal{P}(z)=\left\{Q_{j}: j \in I(z)\right\}$ and define the function $\mu:[0,1) \rightarrow \mathbb{R}$ by

$$
\mu(z)=\sup _{a_{j}>0}\left\{\inf _{x>z}\left(x-\sum_{j \in I(z)} a_{j} \log \left|Q_{j}(x)\right|\right)\right\} .
$$

If $\alpha$ is a totally positive algebraic integer such that $Q_{j}(\alpha) \neq 0$ for $j \in I(z)$, then

$$
\operatorname{tr}(\alpha) \geq \mu\left(\alpha_{1}\right) \text {. }
$$

The only totally positive algebraic integers for which inequality (5) can fail are the roots of the polynomials $Q_{j}, 1 \leq j \leq 91$, and their translates by positive integers. The theorem will be proved once we establish the following lemma.

\section{Lemma.}

$$
\mu(z)>\max (1.780022,1.66+z), \quad 0 \leq z<1 .
$$

Proof. To prove inequality (1) it is enough to estimate $\mu(0)$. We found a set of 24 polynomials $\mathcal{P}(0)=\left\{Q_{j}: j \in I(0)\right\}$ and 24 numbers $a_{j}>0, j \in I(0)$, such that

$$
x-\sum_{j \in I(0)} a_{j} \log \left|Q_{j}(x)\right|>1.780022 \quad \forall x>0 .
$$

The indexes in $I(0)$ and the values of the $a_{j}$ are given in Table 3 at the end of this paper.

To prove that $\mu(z)>1.66+z$, we construct a partition $z_{0}=0<z_{1}<\cdots<$ $z_{20}<z_{21}=1$ of $[0,1]$ into 21 subintervals such that $\mu\left(z_{i}\right)>1.66+z_{i+1}, 1 \leq i \leq 20$, and $\mu(0)>1.780022>1.66+z_{1}$. Since $\mu$ is nondecreasing, the lemma follows. In the last section we explain how we found the $z_{i}$.

Remark. The values if $z_{i}$ and the corresponding indexes $I\left(z_{i}\right)$ are listed in Table 2 at the end of this paper. The complete data for the computation of $\mu\left(z_{i}\right)$ can be downloaded as a PDF document from www.ehu.es/mat/pdf/DataAlgInt.pdf. 
Remark. There are only 14 exceptions to inequality (1), the roots of $Q_{i}$ for $i=$ $2,5,12,25,26$. As for inequality (2), the exceptions are the roots of $Q_{i}$ for $i=$ $2,5,7,12,18,22,25,26,31$ (for a total of 26), and those obtained from them by adding a positive integer.

TABLE 1. Polynomials $Q_{i}$

\begin{tabular}{|c|c|}
\hline$i$ & $\overline{Q_{i}}$ \\
\hline 1 & $x$ \\
\hline 2 & $-1+x$ \\
\hline 3 & $-2+x$ \\
\hline 4 & $-3+x$ \\
\hline 5 & $1-3 x+x^{2}$ \\
\hline 6 & $1-4 x+x^{2}$ \\
\hline 7 & $2-4 x+x^{2}$ \\
\hline 8 & $2-5 x+x^{2}$ \\
\hline 9 & $3-5 x+x^{2}$ \\
\hline 10 & $5-5 x+x^{2}$ \\
\hline 11 & $7-6 x+x^{2}$ \\
\hline 12 & $-1+6 x-5 x^{2}+x^{3}$ \\
\hline 13 & $-1+5 x-6 x^{2}+x^{3}$ \\
\hline 14 & $-1+7 x-6 x^{2}+x^{3}$ \\
\hline 15 & $-1+8 x-6 x^{2}+x^{3}$ \\
\hline 16 & $-2+8 x-6 x^{2}+x^{3}$ \\
\hline 17 & $-1+9 x-6 x^{2}+x^{3}$ \\
\hline 18 & $-3+9 x-6 x^{2}+x^{3}$ \\
\hline 19 & $-4+11 x-7 x^{2}+x^{3}$ \\
\hline 20 & $-5+12 x-7 x^{2}+x^{3}$ \\
\hline 21 & $-5+13 x-7 x^{2}+x^{3}$ \\
\hline 22 & $-7+14 x-7 x^{2}+x^{3}$ \\
\hline 23 & $-10+18 x-8 x^{2}+x^{3}$ \\
\hline 24 & $-13+19 x-8 x^{2}+x^{3}$ \\
\hline 25 & $1-7 x+13 x^{2}-7 x^{3}+x^{4}$ \\
\hline 26 & $1-8 x+14 x^{2}-7 x^{3}+x^{4}$ \\
\hline 27 & $1-8 x+15 x^{2}-8 x^{3}+x^{4}$ \\
\hline 28 & $1-8 x+16 x^{2}-8 x^{3}+x^{4}$ \\
\hline 29 & $2-12 x+18 x^{2}-8 x^{3}+x^{4}$ \\
\hline 30 & $9-28 x+26 x^{2}-9 x^{3}+x^{4}$ \\
\hline 31 & $11-31 x+27 x^{2}-9 x^{3}+x^{4}$ \\
\hline 32 & $13-37 x+32 x^{2}-10 x^{3}+x^{4}$ \\
\hline 33 & $14-38 x+32 x^{2}-10 x^{3}+x^{4}$ \\
\hline 34 & $15-39 x+32 x^{2}-10 x^{3}+x^{4}$ \\
\hline 35 & $16-41 x+33 x^{2}-10 x^{3}+x^{4}$ \\
\hline 36 & $17-42 x+33 x^{2}-10 x^{3}+x^{4}$ \\
\hline 37 & $19-45 x+34 x^{2}-10 x^{3}+x^{4}$ \\
\hline 38 & $-1+10 x-25 x^{2}+22 x^{3}-8 x^{4}+x^{5}$ \\
\hline
\end{tabular}


TABle 1. Polynomials $Q_{i}$ (continued)

\begin{tabular}{|c|c|}
\hline 39 & $-1+11 x-29 x^{2}+26 x^{3}-9 x^{4}+x^{5}$ \\
\hline 40 & $-1+12 x-31 x^{2}+27 x^{3}-9 x^{4}+x^{5}$ \\
\hline 41 & $-1+13 x-32 x^{2}+27 x^{3}-9 x^{4}+x^{5}$ \\
\hline 42 & $-1+15 x-35 x^{2}+28 x^{3}-9 x^{4}+x^{5}$ \\
\hline 43 & $-1+10 x-30 x^{2}+29 x^{3}-10 x^{4}+x^{5}$ \\
\hline 44 & $-1+11 x-34 x^{2}+31 x^{3}-10 x^{4}+x^{5}$ \\
\hline 45 & $-1+11 x-35 x^{2}+31 x^{3}-10 x^{4}+x^{5}$ \\
\hline 46 & $-3+20 x-41 x^{2}+32 x^{3}-10 x^{4}+x^{5}$ \\
\hline 47 & $-1+12 x-40 x^{2}+33 x^{3}-10 x^{4}+x^{5}$ \\
\hline 48 & $-3+20 x-42 x^{2}+33 x^{3}-10 x^{4}+x^{5}$ \\
\hline 49 & $-3+21 x-43 x^{2}+33 x^{3}-10 x^{4}+x^{5}$ \\
\hline 50 & $-4+25 x-47 x^{2}+34 x^{3}-10 x^{4}+x^{5}$ \\
\hline 51 & $-5+29 x-51 x^{2}+35 x^{3}-10 x^{4}+x^{5}$ \\
\hline 52 & $-7+36 x-61 x^{2}+41 x^{3}-11 x^{4}+x^{5}$ \\
\hline 53 & $-9+41 x-64 x^{2}+41 x^{3}-11 x^{4}+x^{5}$ \\
\hline 54 & $-11+49 x-72 x^{2}+43 x^{3}-11 x^{4}+x^{5}$ \\
\hline 55 & $-17+66 x-89 x^{2}+50 x^{3}-12 x^{4}+x^{5}$ \\
\hline 56 & $-53+144 x-141 x^{2}+63 x^{3}-13 x^{4}+x^{5}$ \\
\hline 57 & $-64+175 x-168 x^{2}+72 x^{3}-14 x^{4}+x^{5}$ \\
\hline 58 & $-66+176 x-168 x^{2}+72 x^{3}-14 x^{4}+x^{5}$ \\
\hline 59 & $-67+177 x-168 x^{2}+72 x^{3}-14 x^{4}+x^{5}$ \\
\hline 60 & $-67+185 x-174 x^{2}+73 x^{3}-14 x^{4}+x^{5}$ \\
\hline 61 & $-73+190 x-175 x^{2}+73 x^{3}-14 x^{4}+x^{5}$ \\
\hline 62 & $-89+210 x-183 x^{2}+74 x^{3}-14 x^{4}+x^{5}$ \\
\hline 63 & $1-12 x+45 x^{2}-67 x^{3}+42 x^{4}-11 x^{5}+x^{6}$ \\
\hline 64 & $1-12 x+46 x^{2}-68 x^{3}+42 x^{4}-11 x^{5}+x^{6}$ \\
\hline 65 & $1-13 x+47 x^{2}-68 x^{3}+42 x^{4}-11 x^{5}+x^{6}$ \\
\hline 66 & $1-13 x+50 x^{2}-72 x^{3}+43 x^{4}-11 x^{5}+x^{6}$ \\
\hline 67 & $1-14 x+55 x^{2}-74 x^{3}+43 x^{4}-11 x^{5}+x^{6}$ \\
\hline 68 & $1-15 x+59 x^{2}-78 x^{3}+44 x^{4}-11 x^{5}+x^{6}$ \\
\hline 69 & $1-18 x+63 x^{2}-79 x^{3}+44 x^{4}-11 x^{5}+x^{6}$ \\
\hline 70 & $1-11 x+42 x^{2}-67 x^{3}+45 x^{4}-12 x^{5}+x^{6}$ \\
\hline 71 & $1-12 x+48 x^{2}-75 x^{3}+48 x^{4}-12 x^{5}+x^{6}$ \\
\hline 72 & $5-38 x+96 x^{2}-103 x^{3}+52 x^{4}-12 x^{5}+x^{6}$ \\
\hline 73 & $13-73 x+149 x^{2}-138 x^{3}+62 x^{4}-13 x^{5}+x^{6}$ \\
\hline 74 & $19-96 x+177 x^{2}-151 x^{3}+64 x^{4}-13 x^{5}+x^{6}$ \\
\hline 75 & $-1+14 x-66 x^{2}+136 x^{3}-131 x^{4}+61 x^{5}-13 x^{6}+x^{7}$ \\
\hline 76 & $-1+14 x-67 x^{2}+138 x^{3}-132 x^{4}+61 x^{5}-13 x^{6}+x^{7}$ \\
\hline 77 & $-1+15 x-71 x^{2}+142 x^{3}-133 x^{4}+61 x^{5}-13 x^{6}+x^{7}$ \\
\hline 78 & $-1+14 x-67 x^{2}+140 x^{3}-135 x^{4}+62 x^{5}-13 x^{6}+x^{7}$ \\
\hline 79 & $-1+14 x-68 x^{2}+142 x^{3}-136 x^{4}+62 x^{5}-13 x^{6}+x^{7}$ \\
\hline 80 & $-1+15 x-71 x^{2}+144 x^{3}-136 x^{4}+62 x^{5}-13 x^{6}+x^{7}$ \\
\hline 81 & $-1+15 x-72 x^{2}+146 x^{3}-137 x^{4}+62 x^{5}-13 x^{6}+x^{7}$ \\
\hline 82 & $-1+15 x-73 x^{2}+147 x^{3}-137 x^{4}+62 x^{5}-13 x^{6}+x^{7}$ \\
\hline
\end{tabular}


TABle 1. Polynomials $Q_{i}$ (continued)

\begin{tabular}{|c|c|}
\hline 83 & $-1+15 x-75 x^{2}+153 x^{3}-142 x^{4}+63 x^{5}-13 x^{6}+x^{7}$ \\
\hline 84 & $-1+16 x-78 x^{2}+157 x^{3}-143 x^{4}+63 x^{5}-13 x^{6}+x^{7}$ \\
\hline 85 & $-1+17 x-81 x^{2}+158 x^{3}-143 x^{4}+63 x^{5}-13 x^{6}+x^{7}$ \\
\hline 86 & $-1+17 x-82 x^{2}+159 x^{3}-143 x^{4}+63 x^{5}-13 x^{6}+x^{7}$ \\
\hline 87 & $-2+24 x-102 x^{2}+189 x^{3}-166 x^{4}+71 x^{5}-14 x^{6}+x^{7}$ \\
\hline 88 & $1-15 x+83 x^{2}-220 x^{3}+303 x^{4}-220 x^{5}+83 x^{6}-15 x^{7}+x^{8}$ \\
\hline 89 & $1-24 x+206 x^{2}-813 x^{3}+1662 x^{4}-1920 x^{5}+1320 x^{6}$ \\
& $-549 x^{7}+135 x^{8}-18 x^{9}+x^{10}$ \\
\hline 90 & $1-19 x+145 x^{2}-575 x^{3}+1289 x^{4}-1683 x^{5}+1289 x^{6}$ \\
& $-575 x^{7}+145 x^{8}-19 x^{9}+x^{10}$ \\
\hline 91 & $-1+26 x-279 x^{2}+1625 x^{3}-5702 x^{4}+12694 x^{5}-18464 x^{6}$ \\
& $+17848 x^{7}-11549 x^{8}+4980 x^{9}-1401 x^{10}+245 x^{11}$ \\
& $-24 x^{12}+x^{13}$ \\
\hline
\end{tabular}

\section{About the computations}

In this section we comment on how we carried out the computations. All were done using Mathematica ${ }^{\circledR}$ on a Power Macintosh G4 at 400 Ghz.

3.1. The $z_{i}$. We found the points in the partition used to prove the inequality $\mu(z)>1.66+z$ recursively. The first step was to find $z_{20}$ as the smallest value of $z$ such that $\mu(z)>2.66$. In FRS one finds $\mu(0.91)>2.6615$. Starting from here and adding new polynomials to those used in [FRS, we were able to get $\mu(0.8878)>2.66$. This process is iterated as follows: Once $z_{i+1}$ has been found, we look for $z_{i}$ as the smallest $z$ such that $\mu(z)>1.66+z_{i+1}$. The computations are started using all the polynomials in $\mathcal{P}\left(z_{i+1}\right)$ together with new ones chosen according to the heuristics in the Introduction.

3.2. The function $\Lambda$. To compute the function $\Lambda$ defined by equation (4) we use the symbolic capabilities and the high precision routines of Mathematica ${ }^{\circledR}$. For the sake of simplicity, let us assume that we have selected a set of irreducible polynomials $\left\{q_{1}, \ldots, q_{J}\right\} \subset \mathbb{Z}[x]$ with all their roots real and nonnegative, and let $d$ be the sum of the degrees of the $q_{j}$. Given $a_{j}>0,1 \leq j \leq J$, we want to calculate

$$
\Lambda\left(a_{1}, \ldots, a_{J}\right)=\inf _{x>z} f(x), \text { where } f(x)=x-\sum_{j=1}^{J} a_{j} \log \left|q_{j}(x)\right| .
$$

The function $f$ is unbounded on a neighbourhood of the roots of the $q_{j}$ and at infinity. Hence, it has $d$ local minima, which are found solving the equation $f^{\prime}(x)=0$. For this we compute

$$
g(x)=\left(\prod_{j=1}^{J} q_{j}\right)\left(1-\sum_{j=1}^{J} a_{j} \frac{q_{j}^{\prime}}{q_{j}}\right) .
$$

The function $g$ is a polynomial of degree $d$ with $d$ positive roots $\left\{\xi_{1}, \ldots, \xi_{d}\right\}$, each corresponding to a local minimum of $f$. We compute them with the command NSolve $[\mathrm{g}[\mathrm{x}]==0, \mathrm{prec}]$, where prec, the number of significative digits, depends on $d$. Then $\Lambda\left(a_{1}, \ldots, a_{J}\right)=\min _{\xi_{k}>z} f\left(\xi_{k}\right)$. This is the more time consuming part of the computations, especially for large values of $d$. The most difficult case 
corresponds to the evaluation of $\mu(0)$; then $d=110$, prec $=80$ and it took 196 seconds to compute $\Lambda$.

3.3. The optimization. To maximize $\Lambda\left(a_{1}, \ldots, a_{J}\right)$ for $a_{j}>0$, we use two optimization techniques. The first one is a variant of the second Remes algorithm $[\mathrm{R}]$. As observed above, the function $f$ has $d$ local minima $\left\{\xi_{1}, \cdots, \xi_{d}\right\}$, and in general the values of $f\left(\xi_{j}\right)$ will be different. By analogy with the problem of uniform polynomial or rational approximation, we expect that when the maximum value of $\Lambda$ is achieved, there will be a set of $J$ local minima at which $f$ takes precisely that value. Our implementation of the algorithm is as follows:

(1) Start with a given set of coefficients $a_{1}, \ldots, a_{J}$, and find the local minima $\left\{\xi_{1}, \cdots, \xi_{d}\right\}$ of $f$.

(2) Among the $\left\{\xi_{k}: 1 \leq k \leq J\right\}$ such that $\xi_{k}>z$, select the $J+1$ points $\left\{\eta_{1}, \ldots, \eta_{J+1}\right\}$ at which $f$ takes the smallest values. They are called control points.

(3) Solve the linear system of $J+1$ equations in the $J+1$ unknowns $\bar{a}_{j}$ and $\delta$,

$$
\eta_{k}-\sum_{j=1}^{J} \bar{a}_{j} \log \left|q_{j}\left(\eta_{k}\right)\right|=\delta, \quad 1 \leq k \leq J+1 .
$$

(4) Go back to step (1) with $\bar{a}_{1}, \ldots, \bar{a}_{J}$ as new values of the coefficients.

Most of the times three to four iterations are sufficient to get a solution. However, sometimes the algorithm does not give an answer. It may happen for instance that one of the coefficients $\bar{a}_{j}$ is negative. Then we turn to a different method.

The first steps of the second optimization algorithm are the same as above, except that now we choose only $J$ control points, and order them in such a way that $f\left(\eta_{1}\right) \leq f\left(\eta_{2}\right) \leq \cdots \leq f\left(\eta_{J}\right)$. Then we solve $3^{5}$ linear systems of $J$ equations in the variables $\bar{a}_{j}$

$$
\eta_{k}-\sum_{j=1}^{J} \bar{a}_{j} \log \left|q_{j}\left(\eta_{k}\right)\right|=f\left(\eta_{k}\right)+\epsilon_{k}, \quad 1 \leq k \leq J,
$$

where

$$
\epsilon_{k}= \begin{cases}\epsilon, 0 \text { or }-\epsilon & \text { if } 1 \leq k \leq 5, \\ 0 & \text { if } 5<k \leq J,\end{cases}
$$

and $\epsilon>0$ is a small parameter $\left(10^{-6}\right.$ at the beginning of the algorithm). Among the solutions, we select the one that gives the largest value of $\Lambda\left(\bar{a}_{1}, \ldots, \bar{a}_{J}\right)$. If this is greater than $\Lambda\left(a_{1}, \ldots, a_{J}\right)$, we repeat the process with the new coefficients and a larger value of $\epsilon$. Otherwise, we repeat the computations with the original coefficients and a smaller value of $\epsilon$. In practice, since computing $3^{5}$ times the function $\Lambda$ takes too long, we maximize an approximate function

$$
\Lambda^{*}\left(\bar{a}_{1}, \ldots, \bar{a}_{J}\right)=\min _{\xi_{k}>z}\left(\xi_{k}-\sum_{j=1}^{J} \bar{a}_{j} \log \left|q_{j}\left(\xi_{k}\right)\right|\right) .
$$

In order to solve the systems efficiently, we use the routines LUDecomposition and LUBackSubstitution. 
TABLE 2. Values of $z_{i}$ such and sets of indexes $I\left(z_{i}\right)$

\begin{tabular}{|l|l|}
\hline$z_{i}$ & Indexes in $I\left(z_{i}\right)$ \\
\hline \hline 0.0000 & $1,2,3,5,6,7,12,15,17,25,26,39,40,41,42,65,68,69,83,84,85,86,88,89$ \\
\hline 0.1200 & $1,2,3,5,6,12,15,17,25,26,38,39,40,63,65,66,67,77,80,81,82,84$ \\
\hline 0.1284 & $1,2,3,5,6,7,12,15,25,26,38,39,40,47,63,64,66,76,78,81,82,91$ \\
\hline 0.1379 & $1,2,3,5,6,7,12,15,25,26,38,39,47,63,64,75,76,78,82,83,91$ \\
\hline 0.1469 & $1,2,5,12,25,26,38,44,45,47,63,64,75,76,78,79,82,88,91$ \\
\hline 0.1602 & $1,2,5,6,12,13,14,25,26,28,45,64,88$ \\
\hline 0.1862 & $2,3,5,6,12,13,25,26,27,28,43,64,71,90$ \\
\hline 0.2236 & $2,3,5,6,12,13,25,29,46,49,70,87$ \\
\hline 0.2632 & $2,3,5,6,7,12,13,16,18,25,46,48,50,51,72$ \\
\hline 0.3027 & $2,3,5,7,12,13,16,18,25,46,48,51$ \\
\hline 0.3542 & $2,3,5,7,13,16,18,25,52$ \\
\hline 0.4305 & $2,3,5,7,8,18,21,31,53,54,73,74$ \\
\hline 0.4609 & $2,3,5,7,13,18,19,21,30,53,54,73,74$ \\
\hline 0.5074 & $2,3,5,7,18,21,30,31,53,73,74$ \\
\hline 0.5412 & $2,3,7,19,30,31,53,73,74$ \\
\hline 0.5703 & $2,3,7,19,20,22,31,33,53$ \\
\hline 0.6209 & $2,3,7,9,20,22,31,32,33,55$ \\
\hline 0.6803 & $2,3,4,7,9,22,31,33,34,35,42,55$ \\
\hline 0.7243 & $2,3,4,10,22,34,35,36,60$ \\
\hline 0.8008 & $2,3,4,10,22,23,36,37,57$ \\
\hline 0.8878 & $2,3,4,10,11,24,56,58,59,61,62$ \\
\hline
\end{tabular}

TABLE 3. Coefficients used in the computation of $\mu(0)$

\begin{tabular}{|r|r||r|r||r|r|}
\hline$i$ & $a_{i}$ & $i$ & $a_{i}$ & $i$ & $a_{i}$ \\
\hline \hline 1 & 0.557759018834 & 17 & 0.003606632908 & 68 & 0.002310519546 \\
2 & 0.517434403724 & 25 & 0.030260150191 & 69 & 0.003689991355 \\
3 & 0.084850396449 & 26 & 0.028691767226 & 83 & 0.000649281270 \\
5 & 0.198286275885 & 39 & 0.012740975114 & 84 & 0.004596641527 \\
6 & 0.021810634685 & 40 & 0.014443618843 & 85 & 0.003440013771 \\
7 & 0.015104653966 & 41 & 0.005890655111 & 86 & 0.001631544175 \\
12 & 0.086757831518 & 42 & 0.016499770947 & 88 & 0.004321000270 \\
15 & 0.009979630762 & 65 & 0.000839860439 & 89 & 0.002111633764 \\
\hline
\end{tabular}

\section{REFERENCES}

[A] Aparicio, B. On the Asymptotic Structure of the Polynomials of Minimal Diophantine Deviation from Zero, Journal of Approximation Theory 55 (1988), 270-278. MR0968933 (90b:41010)

[BE] Borwein, P. and Erdelyi, T. The integer Chebyshev problem, Math. Comp. 214 (1996), 661-681. MR:1333305 (96g:11077)

[FRS] Flammang, V., Rhin, G., and Smyth, C.J. The integer transfinite diameter of intervals and totally real algebraic integers, J. Theor. Nombres-Bordeaux 9 (1997), 137-168. MR1469665 (98g:11119)

[HS] Habsieger, L. and Salvy, B. On integer Chebyshev polynomials, Math. Comp. 218 (1997), 763-770. MR.1401941 (97f:11053)

[R] Ralston, A First Course in Numerical Analysis, Mc Graw-Hill, New York, 1965. MR0191070 $(32: 8479)$

[Si] Siegel, C.L. The trace of totally positive and real algebraic integers, Ann. Math. 46 (1945), 302-312. MR0012092 (6:257a) 
[Sm1] Smyth, C.J. Totally positive algebraic integers of small trace, Ann. Inst. Fourier Grenoble 34 (1984), 1-28. MR0762691 (86f:11091)

[Sm2] Smyth, C.J. The mean values of totally real algebraic integers, Math. of Comp. 42 (1984), 663-681. MR.0736460 (86e:11115)

[Sm3] Smyth, C.J. An inequality for polynomials, CRM Proceedings and Lecture Notes 19 (1999), 315-321. MR 1684612 (2000d:11145)

Departamento de Matemáticas, Universidad del País Vasco, Aptdo. 644, 48080 Bilbao, SPAIN

E-mail address: mtpagesj@lg.ehu.es

Departamento de Economía Aplicada I, Universidad del País Vasco, Avda. Lehendakari Aguirre 83, 48015 BilbaO, Spain

E-mail address: elpbillm@bs.ehu.es

Departamento de Matemáticas, Universidad del País Vasco, Aptdo. 644, 48080 Bilbao, SPAIN

E-mail address: mtppealj@lg.ehu.es 